\title{
Quality of life of caregivers with relatives suffering from mental illness in Hong Kong: roles of caregiver characteristics, caregiving burdens, and satisfaction with psychiatric services
}

Daniel Fu Keung Wong ${ }^{1 *}$, Angus Yuk Kit Lam', Sau Kam Chan² and Shuk Fan Chan ${ }^{2}$

\begin{abstract}
Background: The study attempted to explore the quality of life (QoL) of Chinese caregivers with mentally ill relatives. It also aimed to examine the differential roles of caregiving burdens, caregiver characteristics, and satisfaction with psychiatric services in caregivers' QoL.

Methods: 276 caregivers with relatives attending community psychiatric facilities in Hong Kong were invited to fil out a questionnaire. One sample t-tests were conducted to compare the results of this study with that of other Chinese populations in Hong Kong, Taiwan, and mainland China. A hierarchical regression analysis was performed to examine the relative influence of different factors on caregivers' QoL.

Results: Our sample of caregivers had significantly lower QoL scores than other Chinese populations. Results also suggest that Chinese caregivers who had chronic illness, younger in age, a lower education level, experienced more difficulties in handling negative symptoms, and were more dissatisfied with mental health services had poorer quality of life. Indeed, caregiver characteristics displayed a much stronger association with caregivers' QoL than did caregiving burdens and satisfaction with psychiatric services.

Conclusions: This study supports the strong association of caregiver characteristics and the QoL of caregivers and establishes the nature of the relationship between satisfaction with mental health services and caregiver QoL. Implications for future research and practice are discussed.
\end{abstract}

Keywords: Quality of Life, Caregivers characteristics, Caregiving Burden, Satisfaction with psychiatric services, Hong Kong

\section{Introduction}

Caring for a family member with mental illness can be burdensome. Caregivers may experience financial burdens, difficulty handling disruptive behavior and fluctuating emotions that cannot be controlled, a lack of time for personal enjoyment and social engagement, difficulty handling the lack of motivation found in the mentally ill family member, and financial difficulties [1,2]. Evidence of family burdens is also found among caregivers with different ethnic backgrounds such as Chileans [3], Swedes [4], Germans [5], Hong Kong Chinese [6,7], and

\footnotetext{
* Correspondence: dfk.wong@cityu.edu.hk

${ }^{1}$ Applied Social Studies, City University of Hong Kong, Hong Kong SAR Full list of author information is available at the end of the article
}

mainland Chinese [8]. The impact of caring burdens on the psychological wellbeing of caregivers has also been well documented, the results generally suggesting that caregivers suffer from poor psychological health and that some of them may even develop mental illness $[2,6]$.

Although it is commonly acknowledged that caregiving burdens can lead to caregivers with a relative suffering from mental illness experiencing poor quality of life (QoL) [2], few studies have explored this relationship empirically. The few studies that have done so found that family burdens were inversely related to the QoL of caregivers with children suffering from mental health problems [9], mood disorders [10], and schizophrenia 
[4]. Similar studies have also been conducted on caregivers with different ethnic backgrounds, the findings also suggesting that Sudanese caregivers [11] and Kuwaiti caregivers [12] with greater caregiving burdens have poorer QoL. A careful review of the literature identified only one English language article concerning caregivers' burdens and the QoL of Chinese caregivers with a schizophrenic family member [13]. The results suggest that the best QoL predictors for such Chinese family caregivers are physical health and household income. However, the study in question is subject to a number of limitations. Because Chinese caregivers living in different parts of China and the world are exposed to different sets of societal and contextual factors influencing their caring experiences, the findings generated by $\mathrm{Li}$ et al. may not be applicable to Chinese people living in other parts of China and the world [13]. Second, because the samples considered in their study were taken from three hospitals, the caregivers who took part in the survey could have been experiencing a higher level of stress due to their relatives' hospitalization. Lastly, caregivers who are living with their mentally ill family members in the community may have different caregiving burden experiences that affect their QoL in different ways. To the best of our knowledge, no previous study has investigated the caregiving burdens and QoL of Chinese caregivers in Hong Kong. This study intended to explore the quality of life of a group of Chinese caregivers whose mentally ill relatives were living in the communities in Hong Kong; and to examine different factors that might influence caregivers' quality of life. In the literature, there are three sets of factors - (1) caregiving situation; (2) caregiver factors; and (3) environmental factors - that can potentially impact on the QoL of Chinese caregivers with relatives suffering from mental illness in Hong Kong (See Figure 1).

\section{Caregiving situation}

The 'caregiving situation' can be defined as variables related to characteristics of the patient's illness that impact on the QoL of the caregiver. These factors may include the functional status of the patient or the caregiver's perception and evaluation of the care needs of the patient. In the mental health literature, objective and subjective burdens are commonly defined as caregiving situations which affect the QoL of caregivers with relatives suffering from mental illness. The 'objective burden' may be defined as 'all those things that the caregiver and/or his or her family has to do (e.g. helping and supervising), experiences (e.g. disturbed family and/or social relations), or is not allowed to do (e.g. hobbies and work) as a consequence of caregiving [14]. It also includes the tasks of handling the emotional needs of the mentally ill relative [2]. On the other hand, the 'subjective burden' may be defined as the distress experienced by the caregiver in dealing with the objective burden [15], potentially including feeling trapped, guilty, shameful, isolated, and emotionally exhausted [15]. Studies in the literature have suggested that Chinese caregivers (i) experience difficulties managing the negative symptoms presented by relatives with mental illness and handling positive symptoms [6]; (ii) pay high psychological and social costs when caring for their relatives [6]; and (iii) have poor mental health outcomes [6,7]. However, no study has examined the caregiving burdens and QoL of Chinese caregivers in Hong Kong. In this study, 'caregiving burdens' are defined as perceived strains

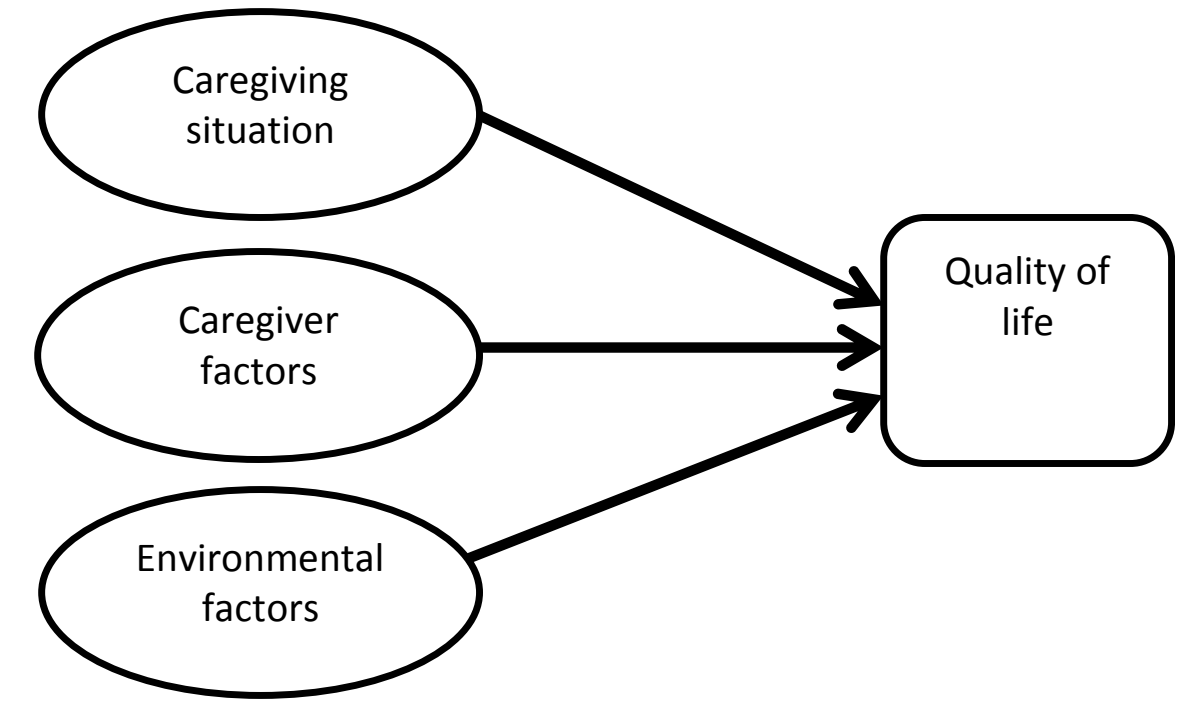

Figure $1 \mathrm{~A}$ model with three sets of factors affecting the QoL of caregivers of family members with mental illness (adapted from the model of White et al., 2004 [14]). 
associated with handling positive and negative symptoms presented by mentally ill relatives and the social and psychological costs borne when caring for such relatives.

\section{Caregiver factors}

According to White et al. [16], caregiver factors are conceptualized as characteristics of the caregiver - who he/she is (i.e. age, gender, etc.) and his/her personal attributes that may impact on the caregiver's QoL. Sales [2] argued that many studies fail to examine contextual variables such as life circumstances and demographic characteristics - that may strongly affect the QoL of caregivers with family member suffering from mental and other chronic illnesses. These demographic characteristics may include the socioeconomic status, ethnicity, age, gender, and family stage and composition of the caregiver. For example, Awadalla et al. [11] found that among caregivers of Sudanese psychiatric patients, those who were females, parents, less educated, older, or had poor physical health were associated with poor QoL. In China, Li, Lambert \& Lambert [13] revealed that among caregivers with schizophrenic relatives, those who had higher household income and better physical health had better QoL. However, because of differences in societal environments, there is uncertainty as to whether the findings of Li et al.'s study are applicable to Chinese populations outside mainland China.

\section{Environmental factors}

Environmental factors include support from family and friends, support from the health care system, and the availability of and satisfaction with environmental resources [16]. Marsh and Johnson [17] maintained that there is a need to pay greater attention to the difficulties caregivers face when dealing with the health care system because caregivers' efforts can place them under tremendous strain and potentially impact on their QoL. However, a review of the literature showed very few empirical studies exploring the impact of environmental factors such as satisfaction with services on the QoL of caregivers with relatives suffering from mental illness. In this study, we attempted to examine the relationship such environmental factors have with QoL among caregivers with relatives suffering from mental illness.

Since previous findings appear to suggest that caregiving burdens are consistently related to poor mental health and QoL among caregivers, one assumption made in this study was that such burdens would have the strongest association with the QoL of caregivers. Our study also hypothesized that caregiver factors and environmental factors are the second and third most important sets of factors associating with the QoL of caregivers. This prioritization was based on the findings relating to each such set of factors reflected in the literature.

\section{Objectives}

This study aimed at explore the quality of life and caregiving burdens of caregivers with relatives suffering from mental illness in Hong Kong; and examining the association between caregiving burdens, caregiver characteristics, satisfaction with mental health services and the QoL of caregivers.

\section{Hypotheses}

There were three hypotheses in this study: (1) caregivers with greater caregiving burdens have poorer quality of life; (2) caregivers with a lower level of satisfaction with mental health services have poorer quality of life; and (3) 'caregiving burdens' had the stronger association with the QoL of caregivers than do 'caregiver factors' and 'satisfaction with psychiatric services'.

\section{Method \\ Participants}

'Caregiver' in this study refers to a person with a mentally ill family member. It is not necessary that the caregiver live with the mentally ill family member, but they have to provide regular support, such as visits and daily care, to the ill relative. 'Ill relative' refers to a person with mental illness. The inclusion criteria were: caregivers (i) aged 18 or above; (ii) able to speak and write simple Chinese; (iii) who provided regular support for a mentally ill relative.

\section{Research design and sampling procedures}

A cross-sectional survey design and a convenience sampling method were adopted for this study. This sampling method was chosen because of the absence of a list of caregivers in Hong Kong and the greater feasibility of obtaining a sample of caregivers through various social service organizations in all 18 Hong Kong districts. Participants were caregivers of individuals with mental illness who were members of the various communitybased mental health service units. The nature of these service units were half-way houses, integrated community mental health services and social clubs. The research team approached the caregivers and gave details of the survey to them. After signing the consent form, the participants were given a self-administered questionnaire. Since some participants were in their old age and might also be illiterate, the research team provided special assistance to facilitate the completion of their questionnaire. The participants were assured of the voluntary nature of the survey and of the fact that declining to participate in the survey would have no adverse effect on their use of services. It took around 35 minutes to complete the questionnaire. This study was endorsed by the Research Ethics Committee of the 
Baptist Oi Kwan Social Services Organization in Hong Kong, which sponsored the project.

\section{Instruments}

The questionnaire contained socio-demographic questions and the following instruments:

\section{Perceived Chronic Strains Scale (Short Form)}

This is a four-point scale denoting the severity of perceived strains experienced by caregivers in their day-today care of their mentally ill relatives in which the responses range from 'Not stressful at all' (1) to 'Very stressful' (4). The original scale consisted of 32 items categorized into four subscales: (i) difficulty managing drug compliance and follow-up; (ii) difficulty managing bizarre and disturbing behavior among individuals with mental illness; (iii) difficulty handling negative symptoms of persons with mental illness; and (iv) social costs associated with the constant care of people with mental illness [6]. The short form of the scale employed in the study had only 16 items. A test of reliability revealed an acceptable level of internal consistency (Cronbach's alpha $=0.933)$.

\section{Satisfaction with Mental Health Services in Hong Kong}

The research team developed this scale to evaluate caregivers' satisfaction with 10 community-based mental health services available to their mentally ill relatives in Hong Kong, such as specialist outpatient clinics and supported employment. This five-point scale ranged from 'Very dissatisfied' (1) to 'Very satisfied' (5), and the caregivers were encouraged to rate only those services that their relatives had used or were using. Examples of questions asked are: were the caregivers satisfied with (i) the opening hours for the services; (ii) the services provided by psychiatrists, nurses, etc.; and (iii) the amount they had to pay? A test of reliability revealed an acceptable level of internal consistency (Cronbach's alpha $=0.867$ to 0.947 ).

World Health Organization Quality of Life Scale - BRIEF Version (HK)

The World Health Organization Quality of Life Scale Brief Version is a 26-item, self-administered questionnaire [18]. Subjects assess their satisfaction with each item in the past 2 weeks on a 5 -point scale (from $1=$ very dissatisfied to $5=$ very satisfied). The 26 items can be divided into four subscales: physical health, psychological health, social relationships, and environmental factors. The scale has well-established psychometric properties and has been widely used in different cultures. The Cronbach's alpha values for this scale and its subscales ranged from 0.684 to 0.810 , indicating an acceptable level of internal consistency.

\section{Results}

Table 1 shows the demographic profile of the participants. The data were drawn from throughout the
Table 1 Demographic characteristics of caregivers ( $\mathbf{n}=$ 276)

\begin{tabular}{|c|c|}
\hline Characteristic & $\%$ \\
\hline \multicolumn{2}{|l|}{ Sex } \\
\hline Male & 20.1 \\
\hline Female & 79.9 \\
\hline \multicolumn{2}{|l|}{ Age } \\
\hline $20-40$ & 8.8 \\
\hline $41-50$ & 16.8 \\
\hline $51-60$ & 37.2 \\
\hline 61-above & 37.2 \\
\hline \multicolumn{2}{|l|}{ Marital status } \\
\hline Single & 8.2 \\
\hline Married & 74.5 \\
\hline Divorced & 4.8 \\
\hline Widowed & 12.5 \\
\hline \multicolumn{2}{|l|}{ Education } \\
\hline Primary or below & 41.5 \\
\hline Secondary & 45.5 \\
\hline University & 13.0 \\
\hline \multicolumn{2}{|l|}{ Employment } \\
\hline Full-time & 23.3 \\
\hline Housewife & 39.7 \\
\hline Student & 1.2 \\
\hline Part-time & 8.2 \\
\hline Unemployed & 3.5 \\
\hline Retired & 24.1 \\
\hline \multicolumn{2}{|l|}{ Income } \\
\hline Social security scheme & 9.2 \\
\hline $5000-20000$ & 57.9 \\
\hline $20001-40000$ & 17.6 \\
\hline 40001 or above & 6.6 \\
\hline Unknown & 8.7 \\
\hline \multicolumn{2}{|l|}{ Relationship } \\
\hline Father & 12.0 \\
\hline Mother & 57.1 \\
\hline Child & 8.7 \\
\hline Sibling & 10.9 \\
\hline Spouse & 11.3 \\
\hline \multicolumn{2}{|l|}{ Years of care } \\
\hline $0-5$ & 29.1 \\
\hline $6-10$ & 26.2 \\
\hline $11-20$ & 26.9 \\
\hline Above 20 & 17.8 \\
\hline \multicolumn{2}{|c|}{ Type of mental illness of the family member } \\
\hline Schizophrenia & 36.1 \\
\hline Early psychosis & 18.6 \\
\hline Delusion & 5.4 \\
\hline Bipolar affective disorder & 12.2 \\
\hline Depression & 13.1 \\
\hline Anxiety disorders & 12.3 \\
\hline Others & 2.2 \\
\hline Living together & \\
\hline
\end{tabular}


territory and covered all 18 administrative districts of Hong Kong. Most of the caregivers who participated in this study were mothers and/or females. The typical age of the caregivers was relatively high, with over $70 \%$ being more than 51 years old. In terms of employment status, the largest groups were housewives and retirees (39.7\% and $24.1 \%$, respectively). About $58 \%$ had monthly family income of between HK\$5000 and HK\$20000 (US $\$ 1=H K \$ 7.80)$. Thirty-six percent of the ill relatives suffered from schizophrenia, while $18.6 \%$ had been diagnosed with early psychosis.

Table 2 displays the caregiving strains experienced by the caregivers. The most highly rated strains experienced by the caregivers were related to 'difficulty handling bizarre and disturbing behavior' and 'difficulty managing fluctuating emotions of ill relatives' ( $\mathrm{M}=$ $2.96, \mathrm{M}=2.96)$. Next on the list of caregiver strains were 'difficulty handling suicidal thoughts/attempts committed by the ill relative' and the issue of the ill relative's lack of employment $(\mathrm{M}=2.83, \mathrm{M}=2.78)$.

Hierarchical regression analysis was performed to test the relative impacts of the three sets of factors (i.e. caregiving situation, caregiver characteristics, and environmental factors) on the caregivers' QoL. In step 1, the caregiving situation (i.e. caregiving burdens) - including 'difficulty handling bizarre behavior and excessive and uncontrollable emotions', 'difficulty handling negative symptoms', and 'social costs associated with the care of a relative with mental illness' - was entered into the first block. Caregiver characteristics such as the year in which the caregiver assumed responsibility for the relative's care, the caregiver's own chronic illness, family income, and the age of the caregiver were entered as the second block. The final block consisted of the environmental factor of 'satisfaction with mental health services'. The results suggest that the Chinese caregivers who had chronic illness, younger in age and a lower education level had poorer quality of life. As a domain, caregiver characteristics explained $12 \%$ of the variance in QoL of Chinese caregivers with relatives suffering from mental illness in Hong Kong (Table 3), (see Figure 2). 'Caregiving burdens' and 'satisfaction with mental health services' also displayed significant association with caregiver's QoL with explained variances of $3 \%$. Of the three subscales for chronic strains, only 'difficulty handling negative symptoms' had significant association with the QoL of the caregivers. In other words, Chinese caregivers who experienced more difficulties in handling negative symptoms and were dissatisfied with mental health services had poorer quality of life. To conclude, the results supported hypothesis 1 and hypothesis 2 , the hypothesis 3 was rejected.

\section{Discussion}

In this study, the quality of life of our caregivers was measured by The World Health Organization Quality of Life Scale - BRIEF Version, which has been used to measure the quality of life of other Chinese populations in Taiwan and mainland China $[13,18,19]$. With the availability of data in the other studies, one sample ttests were performed to examine the differences in QoL scores between Chinese caregivers in the three places [Table 4]. The results indicate that that the caregivers in our sample had significantly poorer QoL than the general public in Hong Kong [18], mainland China [13], and Taiwan [19], and that their QoL was significantly

Table 2 Caregiving burdens of caregivers

\begin{tabular}{ll}
\hline & Mean \\
\hline 1. Bizarre behaviour manifested by the ill relative (e.g. muttering to oneself) & 2.96 \\
2. Fluctuating emotions of the ill relative & 2.96 \\
3. Suicidal thoughts or attempts made by ill relative & 2.83 \\
4. Being unemployed & 2.78 \\
5. III relative refuses to take medication & 2.72 \\
6. Disputes among family members arising from differences on how to handle the ill relative & 2.72 \\
7. Destructive behaviour shown by ill relative (e.g. damaging furniture) & 2.72 \\
8. III relative refuses to go for medical follow-ups & 2.69 \\
9. Household life affected due to care of the person & 2.63 \\
10. Financial difficulties because of having to take care of the ill relative & 2.58 \\
11. III relative neglects personal hygiene & 2.57 \\
12. III relative refuses to go to work & 2.56 \\
13. III relative spends a great deal of time in bed & 2.55 \\
14. III relative idle at home & 2.47 \\
15. Cannot participate in social activities due to care of the ill relative & 2.31 \\
16. III relative refuses to perform household chores & 2.16 \\
Overall mean & 2.09 \\
\hline
\end{tabular}


Table 3 Differential impact of caregiving situation, caregiver characteristics, and environmental factors in influencing QoL of caregivers

\begin{tabular}{|c|c|c|c|c|}
\hline Predictors & Beta & $R^{2}$ & $R^{2}$ change & $F$ change \\
\hline \multicolumn{5}{|l|}{ Step 1} \\
\hline Caregiving situation (Chronic strains) & & 0.03 & 0.03 & 2.53 \\
\hline Difficulty handling bizarre behavior and excessive and uncontrollable emotions & -0.06 & & & \\
\hline Difficulty handling negative symptoms & $-0.20^{*}$ & & & \\
\hline Social costs associated with care & -0.13 & & & \\
\hline \multicolumn{5}{|l|}{ Step 2} \\
\hline Caregivers' characteristics & & 0.15 & 0.12 & 4.78 \\
\hline Year assumed responsibility for care of the relative & -0.05 & & & \\
\hline Caregiver's own chronic illness & $-0.25^{* * *}$ & & & \\
\hline Family income & 0.01 & & & \\
\hline Living together with the relative & -0.02 & & & \\
\hline Age of caregiver & $0.31^{* * *}$ & & & \\
\hline Caregiver's education level & $0.17^{*}$ & & & \\
\hline \multicolumn{5}{|l|}{ Step 3} \\
\hline Environmental factors (Satisfaction with mental health services) & $0.14^{*}$ & 0.18 & 0.03 & 5.31 \\
\hline
\end{tabular}

${ }^{*}<0.05,{ }^{*}<0.01,{ }^{* * *}<0.001$

lower than that of their caregiver counterparts in mainland China [13]. Some of these results should not be surprising, because numerous studies have already found that caregivers of relatives with special needs have lower QoL than the general population [20,21]. Nevertheless, what is surprising is that our group of caregivers had poorer QoL than caregivers in mainland China.
Different expectations on the availability and adequacy of psychiatric services may explain the QoL difference between the two groups. In mainland China, very few community-based psychiatric services are available in the community, and members of the general public are largely expected to care for and support mentally ill relatives themselves. In contrast, there is a high

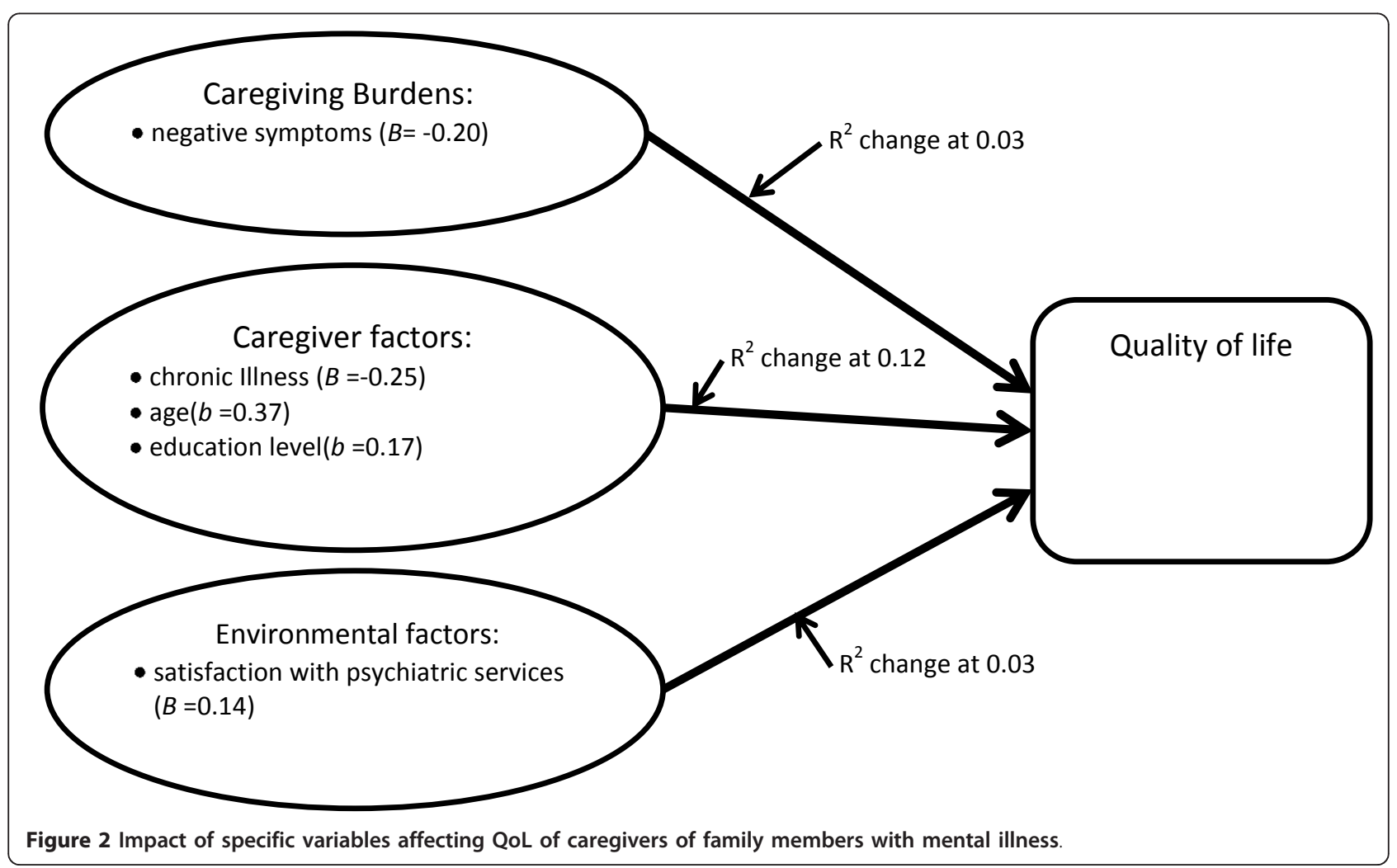


Table 4 A comparison table of QoL scores between our sample of caregivers and those found in Taiwan and China

\begin{tabular}{|c|c|c|c|c|}
\hline \multicolumn{5}{|c|}{ Our sample versus caregivers of relatives with schizophrenia in China $(n=96)$} \\
\hline & Our sample & Caregivers in China & $t$ & $p$ \\
\hline QOL Physical & $13.37(2.40)$ & $14.08(2.36)$ & -4.86 & $<0.001$ \\
\hline QOL Psychological & $12.07(2.55)$ & $12.92(2.68)$ & -5.51 & $<0.001$ \\
\hline QOL Social & $12.87(2.36)$ & $14.44(2.40)$ & -11.09 & $<0.001$ \\
\hline QOL Environmental & $12.61(2.10)$ & $12.32(2.60)$ & 2.29 & 0.023 \\
\hline \multicolumn{5}{|c|}{ Our sample versus Hong Kong general population $(n=155)$} \\
\hline & Our sample & HK general population & & \\
\hline QOL Physical & $13.37(2.40)$ & $15.85(2.13)$ & -17.12 & $<0.001$ \\
\hline QOL Psychological & $12.07(2.55)$ & $14.77(2.39)$ & -17.56 & $<0.001$ \\
\hline QOL Social & $12.87(2.36)$ & $14.26(2.39)$ & -9.82 & $<0.001$ \\
\hline QOL Environmental & $12.61(2.10)$ & $13.74(2.45)$ & -8.96 & $<0.001$ \\
\hline \multicolumn{5}{|c|}{ Our sample versus the general population in China $(n=50)$} \\
\hline & Our sample & China general population $N=50$ & $t$ & $p$ \\
\hline QOL Physical & $13.37(2.40)$ & $15.8(2.9)$ & -16.771 & $<0.000$ \\
\hline QOL Psychological & $12.07(2.55)$ & $14.3(2.5)$ & -14.49 & $<0.000$ \\
\hline QOL Social & $12.87(2.36)$ & $13.7(3.0)$ & -5.87 & $<0.000$ \\
\hline QOL Environmental & $12.61(2.10)$ & $13.2(2.4)$ & -4.68 & $<0.000$ \\
\hline \multicolumn{5}{|c|}{ One sample t-test $p<.05$} \\
\hline \multicolumn{5}{|c|}{ Our sample versus the Taiwanese general population $(n=132,045)$} \\
\hline & Our sample & Taiwan general population & $t$ & $p$ \\
\hline QOL Physical & $13.37(2.40)$ & $15.05(2.08)$ & -11.58 & $<0.000$ \\
\hline QOL Psychological & $12.07(2.55)$ & $13.61(2.27)$ & -10.00 & $<0.000$ \\
\hline QOL Social & $12.87(2.36)$ & $14.39(2.20)$ & -10.74 & $<0.000$ \\
\hline QOL Environmental & $12.61(2.10)$ & $13.00(2.15)$ & -3.09 & $<0.002$ \\
\hline
\end{tabular}

expectation that the government will provide adequate psychiatric services in Hong Kong that invariably heightens caregivers' and consumers' expectations and sometimes leads to dissatisfaction with government involvement in the provision of psychiatric services. Indeed, the government of Hong Kong Special Administrative Region has openly acknowledged that it will uphold the Chinese family tradition of caring for the ill relative, and that it would support these families to take care of the relative who is suffering from a mental illness to live in the community [22]. This difference in people's expectations of the government's role in relation to their own responsibility for caring for ill relatives might affect how they evaluate their own well-being and thus explain the difference in QoL scores between caregivers in Hong Kong and mainland China.

A major objective of this study was to test the relative influences of different sets of factors on the QoL of caregivers with relatives suffering from mental illness. The results indicate that caregivers' characteristics exerted the greatest influence on the QoL of the caregivers, with a total explained variance of $12 \%$. In particular, the caregiver's age, education level, and chronic illness had significant impacts on their QoL. These findings are different from those hypothesized, but serve to support Sales' assertion that "the contextual variables such as life circumstances and demographic characteristics - may strongly affect the QoL of caregivers with [a] family member with chronic illness (including mental illness)" [2]. On the other hand, although caregiving burdens can and do affect the QoL of caregivers, their influence might subside with the passage of time, with some caregivers becoming more adjusted to their caregiving role and acquiring more skills in managing mentally ill relatives. Consequently, they may come to experience or evaluate their caregiving role as less burdensome.

On the other hand, caregiver characteristics are more stable characteristics that are less amenable to change. Indeed, some of these characteristics are unchangeable (e.g. age), and others may follow a deteriorating course (e.g. chronic illness). The literature shows that caregiver characteristics affect the QoL of caregivers in two ways: through their direct and indirect effects. In the case of direct effects, caregiver characteristics affect caregivers' QoL irrespective of the caregiving burdens they experience. For instance, more highly educated caregivers tend to have better jobs, higher salaries, and more resources that can enhance their QoL [11]. However, these characteristics may interact with other factors such as 
caregiving burdens to exert indirect effects on the QoL of caregivers. For example, more highly educated caregivers may be able to use their financial and social resources to help them deal with their caregiving burdens, resulting in better QoL. Indeed, further statistical analysis of our data revealed such a result, showing a significant moderating effect of the interaction between caregiver's education level and caregiving burdens on the QoL of caregivers $(B=0.309, F$ change $=2.80, p=$ 0.05 , with $1 \%$ explained variance). Given the limited availability of evidence concerning the relationship between the characteristics and QoL of caregivers with mentally ill relatives [16], future studies should provide more empirical data to clarify the nature of this relationship.

The second set of variables - caregiver's strains explained 3\% of the variance in the QoL of caregivers. Further analysis showed that only negative symptoms had a significant impact on the QoL of caregivers who participated in this study. These findings echo those findings found in a previous study in Hong Kong [6] and overseas [23]. One possible explanation for these findings is that although positive symptoms may be difficult to manage, they may be perceived as 'part of the illness' beyond the caregiver's control and therefore as symptoms that have to be tolerated and accepted. On the other hand, caregivers may perceive negative symptoms such as 'idle at home' and 'refuse to go to work' as forms of 'laziness' that are amenable to change through personal willpower. This attitude may in fact be rooted in the Chinese belief in Confucian work ethics emphasizing hard work, perseverance, and patience. Simply put, in Chinese culture, there is a strong focus on one's productivity, and anyone who does not work and remains at home is likely to be considered lazy and unproductive [24]. Thus, it was not surprising to find that some caregivers might have had difficulty accepting the negative symptoms presented by their ill relatives. Moreover, unlike positive symptoms which may be more amenable to medication, negative symptoms are issues caregivers have to spend time and energy addressing every day. Further studies are needed to clarify whether the above assumptions are correct and to develop strategies on raising Chinese caregivers' awareness of how such attribution may affect their caregiving burdens and quality of life. At present, there is only one government subsidized family resource centre for family members with relatives suffering from mental illness in Hong Kong. However, many community mental health services are also providing psycho-education programmes for family members. It would be interesting to incorporate the above-mentioned cultural values into the psycho-education programmes, and to examine whether an increase in such awareness might reduce the culturally biased perception of negative symptoms found among some Chinese family members with relatives suffering from mental illness.

Turning to environmental factors, satisfaction with mental health services explained 3\% of the variance in the QoL of the caregivers. As noted in the literature review, very few studies have examined the relationship between satisfaction with psychiatric services and caregivers' quality of life. Our study makes a contribution to the literature by providing initial evidence supporting the existence of such a relationship. However, further and larger-scale research should be conducted to provide more concrete data specifying the nature of the relationship between these two variables. Moreover, in the Hong Kong context, studies should also evaluate how certain mental health services do or do not meet the needs of caregivers. This research should have a particular focus on developing our understanding of caregivers' knowledge of the different types of services available and the adequacy and accessibility of such services. The knowledge generated would facilitate the future development of policies and programs encouraging caregivers to utilize formal psychiatric services to their full extent.

\section{Limitations}

This study has a number of limitations. First, although our sample of caregivers came from different districts in Hong Kong, they were not randomly selected. Therefore, the results cannot be generalized to the wider population of Hong Kong caregivers of mentally ill relatives. Second, our Satisfaction with Mental Health Services Scale was self-constructed, and further and more vigorous validation of the scale is needed. Third, there may be other variables such as social support and certain personality attributes in the three domains that influence the QoL of caregivers in Hong Kong. Researchers should consider including these variables in future studies.

\section{Conclusion}

This study had attempted to clarify the roles of a set of factors associated with the QoL of Chinese caregivers in Hong Kong. It is found that caregivers who were younger, had chronic illness, a lower level of education (caregiver's characteristics), experienced more caregiving burdens and had more dissatisfaction with mental health services had poorer QoL. In particular, caregiver characteristics appeared to have the strongest association with caregiver's QoL. Future research should adopt a longitudinal study design to examine the relationship between QoL and these and other selected variables in the caregiver characteristic, caregiving situation and environmental factor domains, and should examine and impact 
of these variables in influencing the QoL of Chinese caregivers with relatives suffering from mental illness.

\section{Author details}

${ }^{1}$ Applied Social Studies, City University of Hong Kong, Hong Kong SAR.

²Baptist Oi Kwan Social Service, Hong Kong SAR.

\section{Authors' contributions}

DFKW is the person who has written the manuscript. AYKL was the coordinator of this research and had done the initial data analysis. SKC and SFC are staff of the agencies who had coordinated the data collection of this research and contributed to the discussion section of this manuscript. All authors read and approved the final manuscript.

\section{Authors' information}

Daniel Fu Keung Wong ${ }^{1}$ is professor of Department of Applied Social Studies, City University of Hong Kong, Hong Kong SAR. Mr. Angus, Yuk Kit Lam $^{1}$ is a research fellow of Department of Applied Social Studies, City University of Hong Kong, Hong Kong SAR. Miss Sau Kam Chan ${ }^{2}$ is the Senior Service Coordinator of Baptist Oi Kwan Social Service, Hong Kong SAR, and Miss Shuk Fan Chan ${ }^{2}$ is in charge of the Resource and Service Centre for the Relative of Ex-Mentally ill People, Baptist Oi Kwan Social Service, Hong Kong SAR

\section{Competing interests}

The authors declare that they have no competing interests.

Received: 10 August 2011 Accepted: 31 January 2012

Published: 31 January 2012

\section{References}

1. Baronet AM: Factors associated with caregiver burden in mental illness: a critical review of the research literature. Clin Psycho Review 1999, 19:819-841.

2. Sales E: Family burden and quality of life. Quality of Life Res 2003, 12:33-41.

3. Caqueo-Urizar A, Gttierrez-Maldonado J: Burden of care in families of patients with schizophrenia. Quality of Life Res 2006, 15:719-724.

4. Foldemo A, Gullberg M: Quality of life and burden in parents of outpatients with schizophrenia. Soc Psychiatry Psychiatr Epidemiol 2005, 40:133-138.

5. Jungbauer J, Wittmund B, Dietrich S, Angermeyer MC: The disregarded caregivers: Spouses of schizophrenic patients and their living situation. Schiz Bulletin 2004, 30:665-675.

6. Wong DFK: Stress factors and mental health of carers with relatives suffering from schizophrenia in Hong Kong: Implications for culturally sensitive practices. British J Soc Work 2000, 30:365-382

7. Wong DFK, Tsui HKP, Pearson V, Chen EYH, Chiu SN: Family burdens, Chinese health beliefs, and the mental health of Chinese caregivers in Hong Kong. Transcultural Psychiatry 2004, 41:497-513.

8. Liu M, Lambert C, Lambert V: Caregiver burden and coping patterns of Chinese parents of a child with a mental illness. Int J Ment Health Nurs 2007, 16:86-95.

9. Guethmundsson $\mathrm{OO}$, Tomasson K: Quality of life and mental health of parents of children with mental health problems. Nordic J of Psychiatry 2002, 56:413-417.

10. Heru AM, Ryan CE, Vlastos K: Quality of life and family functioning in caregivers of relatives with mood disorders. Psychiatric Rehabilitation Journal 2004, 28(1):67-71.

11. Awadalla AW, Ohaeri JU, Salih AA, Tawfig AM: Subjective quality of life of family caregivers of community living Sudanese psychiatric patients. Soc Psychiatry Psychiatr Epidemiol 2005, 40:755-763.

12. Zahid MA, Ohaeri JU: Relationship of family caregiver burden with quality of care and psychopathology in a sample of Arab subjects with schizophrenia. BMC Psychiatry 2010, 10:71

13. Li J, Lambert CE, Limbert VA: Predictors of family caregivers' burden and quality of life when providing care for a family member with schizophrenia in the People's Republic of China. Nursing and Health Sciences 2007, 9:192-198.
14. Schene AH, Tessler RC, Gamache GM: Instruments measuring family or caregiver burden in severe mental illness. Psychiat Epidemiol 1994, 29:228-240.

15. Schene AH, Tessler RC, Gamache GM, Caregiving in severe mental illness: Conceptualization and measurement. In Mental Health Service Evaluation. Edited by: Knudsen HC, Thornicroft G. New York: Cambridge University Press: 1996:296-316.

16. White CL, Lauzon S, Yaffe MJ, Wood-Dauphinee S: Toward a model of quality of life for family caregivers of stroke survivors. Quality of Life Research 2001, 13:625-638.

17. Marsh D, Johnson D: The family experience of mental illness: Implications for intervention. Profess Psychol Res Pract 1997, 28:229-237.

18. Leung KF, Wong WW, Tay MSM, Chu MML, Ng SSW: Development and validation of the interview version of the Hong Kong Chinese WHOQOLBREF. Quality of Life Research 2005, 14:1413-1419.

19. Yau GK, WHOQOL-BREF Taiwan Version Development Group: WHOQOLBREF Taiwan Version-Development and handbook. WHOQOL Taiwan Version Development Group, Taipei, Taiwan; 22004

20. Xiang YT, Weng YZ: Quality of life of Chinese schizophrenia outpatients in Hong Kong: Relationship to sociodemographic factors and symptomatology. ANZ J of Psychiatry 2007, 41:442-449.

21. Xiang YT, Luk ESL, Lai KYC: Quality of life in parents of children with attention-deficit-hyperactivity disorder in Hong Kong. ANZ J of Psychiatry 2009, 43:731-738.

22. Hong Kong Special Administrative Region: Press Release on Mental Health Services., Retrieved from: www.info.gov.hk/gia/general/200906/10/ P200906100160.htm, June 10, 2009.

23. Weisman AG, Nuechterlein KH, Goldstein MJ, Snyder K: Expressed emotion, attributions, and schizophrenia symptom dimensions. J Abnormal Psychol 1998, 107:335-339.

24. Hui CH, Tan CK: Employee motivation and attitudes in the Chinese workforce. In the Handbook of Chinese Psychology. Edited by: Bond $\mathrm{MH}$ Hong Kong: Oxford University Press; 1996:364-378.

doi:10.1186/1477-7525-10-15

Cite this article as: Wong et al:: Quality of life of caregivers with relatives suffering from mental illness in Hong Kong: roles of caregiver characteristics, caregiving burdens, and satisfaction with psychiatric services. Health and Quality of Life Outcomes 2012 10:15.

\section{Submit your next manuscript to BioMed Central and take full advantage of:}

- Convenient online submission

- Thorough peer review

- No space constraints or color figure charges

- Immediate publication on acceptance

- Inclusion in PubMed, CAS, Scopus and Google Scholar

- Research which is freely available for redistribution 